\title{
DEHESAS Y PAISAJES ADEHESADOS EN CASTILLA YLEÓN
}

\author{
José Manuel LLORENTE PINTO \\ Departamento de Geografía. Universidad de Salamanca.
}

Recibido: 24/01/2011

Aceptado: 20/06/2011

RESUMEN: Las dehesas en Castilla y León ocupan alrededor del 5.5\% del territorio regional, concentrándose básicamente en el sector suroccidental, las provincias de Salamanca, Ávila y Zamora. Presentan una notable variedad de sustratos litológicos, y la encina es la especie arbórea más frecuente en estos montes. Son fincas grandes (entre 350 y 400 ha de media) que suelen llevarse en propiedad, y en las que la orientación productiva fundamental es la ganadera, que supone algo más del $75 \%$ de la producción. Su dependencia de las subvenciones es en la actualidad considerable, como ocurre con otros tipos de explotaciones. El engorde de cerdos ibéricos y la cría de ganado vacuno son los aprovechamientos principales, a los que se subordinan la explotación agrícola y la gestión forestal. Paisajísticamente este sistema agrario se caracteriza por el protagonismo de un monte abierto de quercíneas con grados de densidad variables. En los últimos años se ha incrementado notablemente la carga ganadera, y esta circunstancia, unida a otros procesos, está afectando negativamente a los equilibrios internos de estos agrosistemas y a su propia persistencia, a causa fundamentalmente de los problemas relacionados con un estado adecuado del arbolado.

PALABRAS CLAVE: Dehesa, ganadería extensiva, gestión forestal, paisaje, Castilla y León.

\section{DEHESAS AND SAVANNAH-LIKE LANDSCAPES IN CASTILE AND LEON}

ABSTRACT: The "dehesas" of Castile and Leon occupy about 5.5\% of the regional territory, primarily concentrated in the southwestern sector, the provinces of Salamanca, Avila and Zamora. They show a remarkable variety of lithological substrates, and the Holm oak is the most common tree species in these open woodlands. The farms are large (between 350 and 400 ha on average) and the land tenure is majority ownership. In this farms the livestock is the fundamental production, because it represents just over $75 \%$ of income. The dependence on subsidies is now considerable, as with other types of farms. Fattening Iberian pigs and cattle breeding are the main uses, and agricultural and forest uses are subordinate to the grazing. The landscape of this agricultural system is characterized by the prominence of open oak woodland with varying degrees of density. In recent years it has greatly increased the stocking density, and this, coupled with other processes are adversely affecting the internal balance of these agro-ecosystems and their own persistence, primarily because of problems related to a proper state of the trees.

KEY WORDS: Dehesa, extensive livestock grazing, forest management, landscape, Castile and Leon. 


\section{INTRODUCCIÓN}

Un paisaje agrario, como cualquier otra construcción social, no es una entidad estática. Es el resultado o, si se quiere, el reflejo de un equilibrio de fuerzas sociales e institucionales y potencial ecológico que se acaba sustanciando sobre el terreno en función de la influencia que ejercen esas fuerzas en las posibilidades de aprovechamiento o puesta en valor de los recursos naturales que ofrece un lugar determinado en un lapso temporal variable. No todos los paisajes agrarios tienen la misma inercia o la misma persistencia, pero sí es necesario recordar que realidades paisajísticas muy parecidas no siempre obedecen a contextos técnico-económicos similares.

En general, la evolución de la actividad agraria ha supuesto la generación de sistemas agrarios paulatinamente menos maduros, es decir, caracterizados por una mayor simplicidad paisajística, que no es sino el trasunto de una menor complejidad ecológica y estructural, de una menor persistencia en los ciclos internos de materia y de un incremento de las transferencias de energía entre esos agrosistemas y el exterior. Es en este contexto en el que puede sorprender la supervivencia de unos paisajes agrarios perfectamente funcionales, aunque sometidos a notables tensiones, en los que sobresale la presencia del monte con distintos grados de densidad y madurez, en unas formaciones que suponen en principio un compromiso entre la producción y la estabilidad.

Es razonable pensar que el uso del monte o del bosque aclarado en forma de "parque" con diferentes niveles de densidad arbórea o arbustiva fuera característico coyunturalmente en las áreas que se fueron constituyendo en el frente pionero en los procesos de colonización agraria. Y ese modelo se fue consolidando en zonas con suelos no especialmente fértiles, con una presión demográfica baja, con clima semiárido-subhúmedo pero siempre con parada vegetativa estival y con una estructura de la propiedad latifundista (SÁNCHEZ GóMEZ, 1992).

En efecto, en territorios con esas condiciones ambientales mencionadas más arriba y con una ocupación baja, la persistencia del arbolado en diferentes fases de adehesamiento resultaba un complemento adecuado para la actividad ganadera; es verdad que los pastizales desarbolados son globalmente más productivos que los montes adehesados, pero la presencia de estas plantas leñosas aunque reducen la producción de pasto, la diversifican, moderan la estacionalidad de esa producción, limitan las inclemencias que sufre el ganado y además proporcionan ramón todo el tiempo y, en el caso de las quercíneas, un alimento muy energético en una época crítica del año como es el final del otoño y el principio de invierno. 
Tanto es así que el arbolado de quercíneas también ha subsistido en terrenos estrictamente agrícolas, como sobre las hojas de áreas sometidas a rotación al tercio; en estas zonas las hojas que estaban de barbecho o de posío eran pastadas por el ganado, y el aprovechamiento de la bellota suponía un importante suplemento para las diferentes especies e incluso para la propia población en años malos. Hay que tener en cuenta que estos montes, al estar labrados, solían ser los que proporcionaban mayor cantidad y calidad de fruto.

Esta presencia de un arbolado más o menos diseminado sobre terrenos labrados (ver FIGURA 1) o sobre pastizales no acaba en lo mencionado más arriba. En algunos lugares los pastizales semiagostantes de valles y riberas aparecen salpicados de fresnos; estas fresnedas, que pueden incluir o no otros árboles y arbustos (como otros árboles de ribera, diferentes espinos o las propias quercíneas), tienen un significado funcional parecido al resto de los paisajes adehesados, con la salvedad de que en ellas no se produce el aprovechamiento de la montanera. En algunos lugares de esta región estos pastizales salpicados de fresnos constituían la masa común de pueblos de vocación agrícola que habían reservado estos terrenos complicados de laborear antiguamente como zonas guardadas para el mantenimiento de los ganados, especialmente del ganado de labor. Ese era también el sentido de las dehesas boyales; en efecto en los términos que acabaron constituidos por campos abiertos organizados en hojas de cultivo se solía reservar una porción del terreno para el sostenimiento de ese ganado de labor y frecuentemente también para el aprovisionamiento de leñas y maderas a los vecinos. Estos montes adquirieron un aspecto adehesado que no han mantenido en todas las circunstancias, pero que en cualquier caso y desde un punto de vista fisionómico es equiparable al que podemos encontrar en las dehesas típicas.

En otros casos los montes eran bienes de propios, es decir, propiedad de las corporaciones locales que durante siglos los concejos utilizaron como fuentes de rentas y de aprovisionamiento de sus poblaciones, especialmente para el suministro de leña y cisco. Si las condiciones eran las adecuadas, estos montes acabaron adquiriendo pronto una fisionomía de monte ahuecado, y si salieron indemnes de los procesos desamortizadores (RAMOS SANTOS, 2008), aunque mermados en relación con su dimensión original, han podido llegar a nuestros días convertidos frecuentemente en ese caso en espacios de ocio o de esparcimiento para el conjunto de la población. Un buen ejemplo de esto es el del Monte "El Viejo" de Palencia, que ha sido muy bien estudiado (ALARIO TRIGUEROS, et al. 1981).

Nos encontramos así y por lo que acabamos de escribir con paisajes semejantes pero que no responden a unas mismas condiciones fundiarias ni suelen 
tener una presencia equiparable sobre el territorio. Con todas las excepciones que queramos, las dehesas particulares aparecen ocupando de forma bastante continua amplias zonas, mientras que las antiguas dehesas comunales se suelen caracterizar por una presencia puntual, frecuentemente rodeadas de campos que tradicionalmente entraban en las rotaciones de cultivo. En consecuencia, los paisajes del adehesamiento son fruto de un modo de aprovechamiento agrosilvopastoral que no está siempre basado en el mismo tipo de estructura agraria. Así, las dehesas típicas o particulares son explotaciones agrarias caracterizadas paisajísticamente por un monte adehesado que cuentan con unas condiciones determinadas de tamaño y de forma de tenencia, en el sentido de que son fincas grandes y de propiedad particular. Las dehesas boyales originalmente eran un complemento de otras explotaciones, en buena medida agrícolas, y tenían además un carácter comunal o vecinal.

En definitiva, con estas Figura 1: Encinas recién desmochadas sobre primeras líneas queremos una besana labrada aclarar el título de este artículo y enmarcar nuestra exposición. Los paisajes caracterizados por un monte ahuecado son el resultado de una estrategia en la modificación de la naturaleza, es decir, de lo que conocemos como un tipo de agricultura bastante elemental, y por lo mismo ensayada en muchos lugares con soluciones fisionómicas similares o conver-

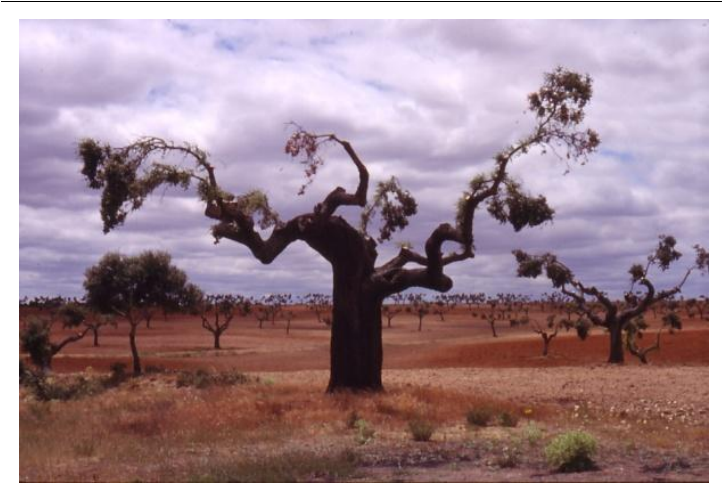

FuENTE: José Manuel Llorente Pinto, Agosto de 1995, Carrascal de Barregas, Salamanca. gentes -una red de árboles diseminados sobre un pastizal o, eventualmente, sobre terrenos cultivados-, aunque sobre formaciones vegetales contrastadas y con modelos de organización de la explotación, de prestaciones de trabajo o de tipos de propiedad o tenencia de la tierra que pueden responder a fórmulas igualmente distintas. Es decir, no todos los sistemas adehesados responden a unas mismas condiciones en términos de la naturaleza jurídica de la propiedad, de las características del régimen de aprovechamiento y de su configuración como explotaciones agrarias.

$\mathrm{Y}$ eso porque esa estrategia que consiste en convertir un monte en un oquedal, que podemos llamar adehesamiento, es una solución bastante elemental; por ello y con las oportunas variantes los sistemas agrosilvopastorales o silvopastorales son una fórmula a la que se ha llegado de manera inde- 
pendiente en diferentes partes del mundo. Por todo ello, es conveniente que definamos con más precisión lo que entendemos por dehesa, "ya que no siempre detrás de un mismo resultado fisionómico -monte ahuecado representado por grandes quercíneas aisladas en un terreno de pasto o alternativamente de pasto y labor- hay un mismo sistema de explotación" (LLORENTE PINTO, 1995: 26).

Las dehesas son explotaciones agropecuarias de cierta complejidad estructural y funcional; son fincas grandes y generalmente de propiedad particular que forman coto redondo y que presentan una orientación productiva fundamentalmente ganadera (LLORENTE PINTO, 1985). Obviamente estas fincas se caracterizan desde el punto de vista paisajístico por el monte adehesado, como señalábamos más arriba, pero la variedad de unidades dentro de una finca si atendemos, por ejemplo, a la densidad del arbolado es considerable, y lo mismo podemos decir si comparamos dos fincas distintas. En el extremo nos podemos encontrar con explotaciones que presentan formas de aprovechamiento perfectamente asimilables a las típicas de la dehesa pero que, por diferentes razones, cuentan con muy poco monte. Esto complica enormemente establecer una definición operativa de esta realidad ${ }^{1}$, y eso probablemente ha dificultado mucho tener información estadística desagregada sobre estas explotaciones.

En este trabajo vamos a centrarnos fundamentalmente en las dehesas que hemos llamado típicas, pero no nos parecía oportuno soslayar de forma absoluta esos otros paisajes adehesados, que, además y dependiendo de las fuentes de información que se utilicen, pueden resultar difíciles de separar o de distinguir de las primeras. Y esto nos lleva a plantear justamente el problema de las fuentes.

\section{FUENTES}

Las fuentes estadísticas convencionales (como los censos o las encuestas sobre la estructura de las explotaciones agrarias) no permiten directamente bien por razones de escala o de diseño o por ambas obtener una información detallada y suficientemente coherente de las explotaciones consideradas desde una óptica de tipología funcional que tenga sus fundamentos en los paisa-

\footnotetext{
${ }^{1}$ En el documento que citamos se propone la siguiente definición de "dehesa" como el "sistema de explotación ganadera y/o cinegética de carácter multifuncional en que al menos el $50 \%$ de la superficie está ocupado por pastizal con arbolado adulto disperso productor de bellotas y con una fracción de cabida cubierta entre el 5 y el 60\%”. Con esta definición que se califica de operativa, se pretende unificar los criterios técnicos y administrativos con la finalidad de crear un registro oficial de este tipo de explotaciones (PULIDO, ET AL., 2010: 5)
} 
jes agrarios. Esto es así porque la información no utiliza como criterio de agrupamiento esa tipología, por otra parte difícil de establecer, y sí más bien las diferentes circunscripciones administrativas, el tamaño de las fincas o una clasificación en función de la orientación técnico-económica que tiene evidentes limitaciones. Esto es todavía más radical en el caso que nos ocupa, por tratarse de explotaciones especialmente complejas tanto desde la perspectiva de los usos del suelo como de las producciones.

Los datos basados en los usos del suelo presentan también imperfecciones, bien se trate de fuentes igualmente de tipo estadístico o de información cartográfica. El problema así no es sólo ni principalmente de naturaleza documental sino más bien conceptual y por esa razón resulta tan complicado cuantificar con alguna precisión la superficie que ocupan en España o en Castilla y León los terrenos adehesados. En definitiva, concretar el significado de dehesa o de paisaje adehesado no es fácil, ya que "dehesa es, ante todo, un término polisémico que se refiere a la vez a un tipo de propiedad, a un tipo de explotación compleja agrícola, pecuaria y forestal, y a una forma particular de cubierta y paisaje fitogeográfico" (Mata Olmo, 2002: 28); y todo esto se complica un poco más por la heterogeneidad que presentan estas fincas, lo que dificulta que se pueda hablar de un modelo o tipo de dehesa.

Para solventar estos problemas hemos recurrido a fuentes variadas que hemos combinado con la intención de recuperar o aprovechar en cada caso la información más valiosa o más pertinente procedente de cada una de ellas. Hay que entender que en unos casos las estadísticas sólo proporcionan datos a escala provincial; cuando la información procede de los usos del suelo, no siempre aparece una clase específica relativa a los montes adehesados y cuando es así la superficie consignada normalmente infravalora la extensión ocupada por las fincas, que no están compuestas exclusivamente por ese tipo de formaciones; en fin, los datos relativos a la estructura agraria generalmente sólo se pueden inferir a partir de una muestra en las zonas en las que se detecta una densidad alta de dehesas.

La información básica de que disponemos sobre la localización y el tamaño de estas fincas procede de las llamadas Fichas de Conservación, que ya hemos presentado en otro lugar (LLORENTE PINTO, 1995). El problema es que no hemos podido encontrar las fichas referidas a toda la región, y sólo las de la provincia de Salamanca (prácticamente completa) y una parte importante de la provincia de Zamora, lo que en cualquier caso supone como mínimo un $80 \%$ de los terrenos adehesados de Castilla y León. Por esta razón y por las características de los propios datos estas fichas se han convertido en la información sobre la que pivota el resto de las fuentes, a pesar de que hayan pasado ya más de 50 años desde la elaboración de estos croquis. 
Los datos que actualmente nos parecen más fiables sobre el reparto provincial de los montes adehesados son los que se han adelantado con los resúmenes del Tercer Inventario Forestal Nacional (http://www.mma.es/ secciones/biodiversidad/inventarios/ifn/ifn3/pdf/estapridac_04_06_2007.pdf); en este documento aparece una categoría que es la de "bosque adehesado" y otra bajo el epígrafe "monte arbolado ralo y disperso". Como ya comentaremos más adelante, esta fuente subestima en general el terreno ocupado por dehesas, pero podemos suponer que al menos mantiene las proporciones; cuando se publiquen los mapas de las distintas provincias, estaremos en condiciones de precisar mucho más en lo relativo a la superficie ocupada por estos montes y a su localización concreta. A esto hay que añadir los datos muy valiosos que proporciona el trabajo de síntesis más ambicioso que se ha hecho en España sobre este tema (MARM, 2008).

Mientras tanto, la fuentes cartográficas de carácter global que vamos a utilizar son dos: el último mapa publicado del proyecto Corine Land Cover, que tiene fecha de 2006 y que puede obtenerse en el centro de descargas del CNIG: (http://centrodedescargas.cnig.es/CentroDescargas/index.jsp), y el Mapa Forestal de España a escala 1/50.000 (MFE50) realizado entre 1997 y 2006 (http://www.mma.es/portal/secciones/biodiversidad/ montes politica forestal/mapa forestal/edicion digital/digital mfe50.htm). La mayor ventaja del mapa del proyecto Corine es que presenta una categoría que en principio alude sin ambigüedades a la realidad que estudiamos; se trata del código 244 , que se refiere a los sistemas agroforestales, y que incluye tanto a los pastizales, prados o praderas con arbolado adehesado, como a los cultivos agrícolas con arbolado adehesado. El MFE50 es un mapa con una información muy rica y llena de matices, pero la extracción de las teselas que pueden considerarse terrenos adehesados no resulta tan obvia. Estas dos informaciones también subestiman para el conjunto de la región la superficie ocupada por dehesas (en especial la primera), al tiempo de incluir terrenos que no parecen característicos de las dehesas típicas; pero con todo nos puede permitir tener una visión de conjunto de la localización de estos terrenos y cuantificar algunos aspectos importantes que extraeremos de otros mapas temáticos.

Por otro lado, la información referida a determinados aspectos de la estructura agraria, a la densidad ganadera o a la composición de la cabaña, la obtendremos del Censo Agrario básicamente, una vez que hayamos establecido los términos municipales en los que hay una densidad apreciable de este tipo de fincas, y a partir del Diagnóstico de las dehesas ibéricas mediterráneas (MARM, 2008). En consecuencia estos resultados no los obtendremos en todos los casos del conjunto de las explotaciones, sino que los inferiremos a partir de los datos municipales de las empresas agrarias de mayor tamaño 
que se encuentran dentro de las áreas con una concentración de dehesas más alta.

\section{LOCALIZACIÓN Y SUPERFICIE OCUPADA POR LOS TERRENOS ADEHESADOS}

Los montes ahuecados de encinas y robles y, en menor medida, quejigos o alcornoques son quizás la seña de identidad más sobresaliente del paisaje rural en el cuadrante suroccidental de la región y ello tanto por su importancia superficial como por su carácter relativamente original dentro de la Submeseta Norte, ya que en el resto de Castilla y León se puede considerar como un tipo de paisaje agrario poco habitual. No puede extrañar así que con frecuencia la primera impresión que causan estos montes es de cierta sorpresa, sobre todo por contraste frente a las dilatadas campiñas prácticamente desarboladas que se han convertido en la imagen estereotipada de Castilla y por la amplitud y continuidad de este paisaje montaraz.

Es así de sobra conocido que es la provincia salmantina la que concentra el mayor número de dehesas. En algunas comarcas de Zamora y Ávila este paisaje también puede ser dominante $\mathrm{o}$, al menos, habitual y además puede presentar cierta continuidad espacial. En el resto de la región, sin embargo, la presencia de dehesas tiene un carácter que podemos calificar de testimonial o puntual y lo mismo podríamos decir de los montes abiertos de quercíneas que no se configuran como una explotación agraria autónoma.

Tabla 1: Superficie ocupada por terrenos adehesados y dehesas (Ha).

\begin{tabular}{|c|c|c|c|c|c|c|}
\hline & \multicolumn{3}{|c|}{ MARM (2008) } & \multirow{2}{*}{$\begin{array}{l}\text { CORINE } \\
\text { Montes } \\
\text { adehesa- } \\
\text { dos }\end{array}$} & \multirow{2}{*}{$\begin{array}{l}\text { MFE50 } \\
\text { Montes } \\
\text { adehesa- } \\
\text { dos }\end{array}$} & \multirow{2}{*}{$\begin{array}{c}\text { Fichas } \\
\text { conserva- } \\
\text { ción }\end{array}$} \\
\hline & $\begin{array}{l}\text { Montes } \\
\text { adehe- } \\
\text { sados }\end{array}$ & $\begin{array}{c}\text { Montes } \\
\text { adehesa- } \\
\text { dos }\end{array}$ & Dehesas & & & \\
\hline Ávila & 41.898 & 42.817 & 77.896 & 41.219 & 32.312 & 51.518 \\
\hline Burgos & 0 & 469 & 914 & 2.651 & 1.241 & 1.420 \\
\hline León & 0 & 225 & 387 & 5.084 & 372 & 1.849 \\
\hline Palencia & 0 & 8 & 10 & 7.139 & 82 & 2.353 \\
\hline Salamanca & 320.808 & 309.840 & 534.503 & 262.465 & 325.493 & 396.728 \\
\hline Segovia & 8.117 & 4.316 & 9.580 & 1.507 & 8.939 & 7.448 \\
\hline Soria & 0 & 1.436 & 2.666 & 1.231 & 2.766 & 1.769 \\
\hline Valladolid & 0 & 1.282 & 2.093 & 3.190 & 1.530 & 1.954 \\
\hline Zamora & 26.058 & 31.928 & 59.358 & 47.509 & 27.052 & 43.152 \\
\hline Total & 396.881 & 392.321 & 687.407 & 391.995 & 399787 & 514.703 \\
\hline
\end{tabular}

FuENTE: Tercer Inventario Forestal Nacional, MARM 2008, Corine Land Cover 2006, Mapa Forestal de España 1/50.000, y Fichas de Conservación. 
A este respecto los datos que nos proporciona el tercer Inventario Forestal Nacional son bastante rotundos, ya que sólo se consignan "bosques adehesados" en cuatro provincias (Salamanca, Ávila, Zamora y Segovia) y una abrumadora mayoría de estos terrenos (algo más del 80\%) se localizan en Salamanca, mientras que a Segovia se le adjudica únicamente el $2 \%$ del total. Esta situación varía algo si se consideran otras fuentes y sobre todo si no sólo se tiene en cuenta el terreno ocupado por formaciones adehesadas, es decir, si se contabiliza también el conjunto de la extensión de las fincas adehesadas. Un resumen de las estimaciones procedentes de las distintas fuentes los presentamos en la TABLA 1 .

Mapa 1: Localización de los terrenos adehesados

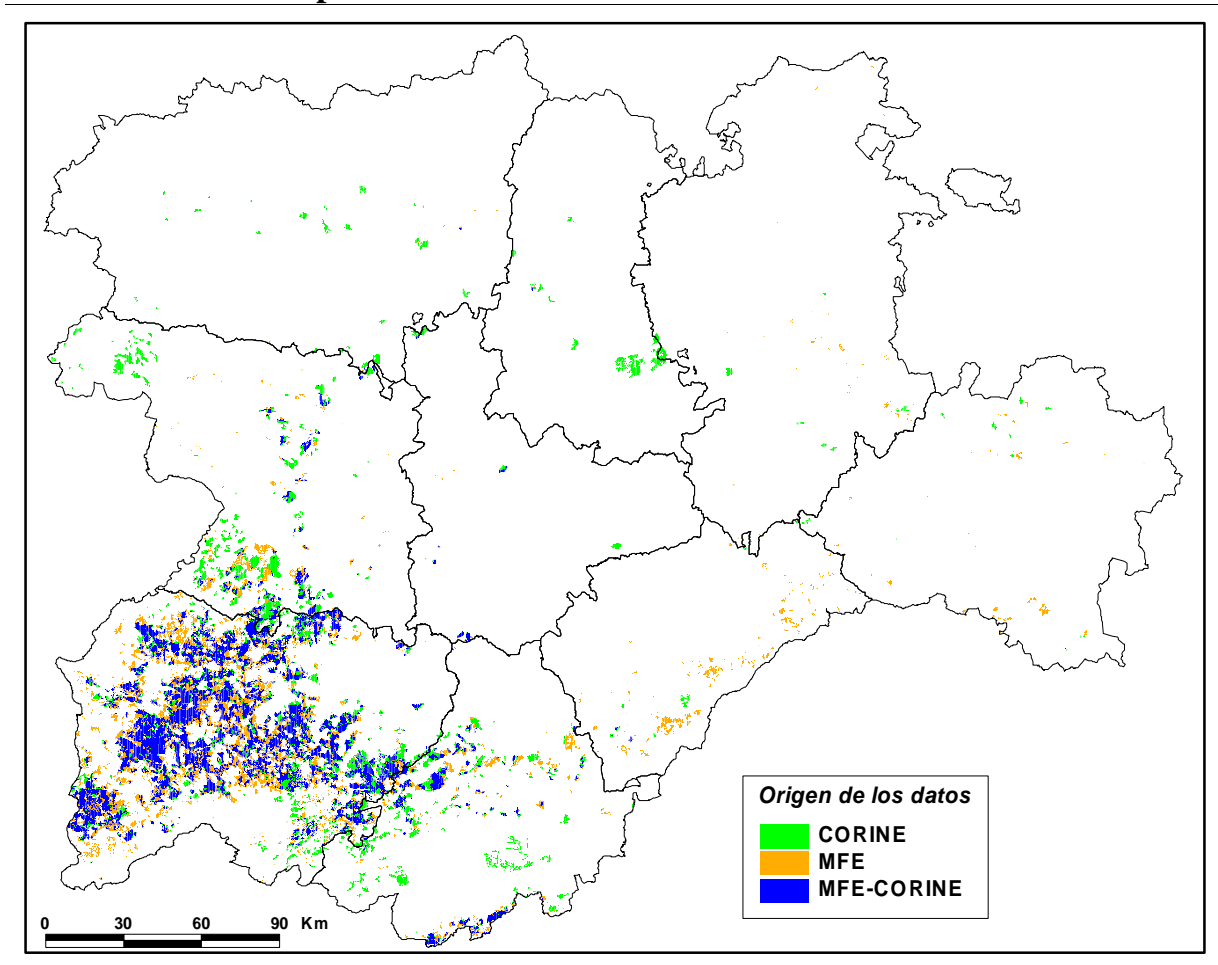

FUENTE: Mapa Forestal de España 1/50.000 y Corine Land Cover, 2006.

En definitiva, las dehesas son algo propio del rincón suroccidental de la región y resultan una rareza fuera de este ámbito. Entre Salamanca, Ávila y Zamora ocupan como mínimo el 89\% de los terrenos adehesados, que significan cerca de 400.000 ha para las cuatro fuentes consideradas, y además con unas desviaciones muy pequeñas. Más llamativas son las diferencias que se aprecian en los valores provinciales La superficie ocupada por montes 
adehesados y otros tipos de terrenos que forman parte de las dehesas (praderas, pastos, matorrales y tierras cultivadas sin árboles o con muy pocos árboles) la hemos evaluado en unas $515.000 \mathrm{Ha}$, es decir, algo así como el 5.5\% de la extensión del territorio regional y alrededor del $15 \%$ de la superficie adehesada nacional.

En el MAPA $n^{\circ} 1$ presentamos la localización concreta de los terrenos adehesados a partir de los datos procedentes del proyecto Corine y del Mapa Forestal de España; en la mayoría de estos terrenos (221.471 ha) hay coincidencia entre las dos fuentes; además una buena parte de las manchas identificadas independientemente aparecen dando continuidad a las comunes o a las fijadas por la otra fuente (eso es así en buena parte de la provincia salmantina, en Sayago, a lo largo de la vertiente norte de la Sierra de Ávila, en los valles del Tiétar y del Esla,...); sin embargo, los resultados resultan más dudosos en otros casos, como los que muestra el proyecto Corine en Sanabria o en el entorno de Gredos; y las discrepancias son notables en León, Palencia y Segovia, en los dos primeros casos por aparente exceso del mapa del proyecto Corine o por defecto del MFE50, y en el caso de Segovia por lo contrario.

\section{RASGOS AGROECOLÓGICOS DE LAS DEHESAS}

La ubicación de estos montes ya proporciona una idea de los condicionantes ambientales que enmarcan el potencial ecológico de las explotaciones adehesadas. No obstante, la diversidad de estos rasgos es mayor de la que en principio cabría esperar a tenor de los arquetipos que nos hemos formado sobre este tipo de paisaje agrario; es verdad por ejemplo que mayoritariamente son las penillanuras labradas sobre el zócalo el sustrato más típico de estas fincas, pero en este aspecto como en otros lo menos habitual no siempre es estrictamente anecdótico.

La altitud media de estos terrenos es de $836.5 \mathrm{~m}$, con un coeficiente de variación de 16.1, lo que significa que, a pesar de una horquilla muy amplia en los valores extremos, la variabilidad de este aspecto se puede considerar moderada, y así el 85\% de la superficie adehesada se encuentra entre los $670 \mathrm{~m}$ de altitud y los $1.000 \mathrm{~m}$. Por el contrario, por encima de los $1.200 \mathrm{~m}$ no encontramos ni el $1 \%$ de la extensión total. Tal vez el caso más singular a este respecto lo constituyen las dehesas del Valle del Tiétar, que forman un conjunto claramente diferenciado del resto por situarse a altitudes notablemente inferiores a los valores medios (el 1.6\% de los terrenos adehesados se sitúan por debajo de los $450 \mathrm{~m}$ ), lo que sin duda hace que guarden más semejanzas con las dehesas del resto de la Submeseta Sur que con las que encontramos al norte del Sistema Central. No hay diferencias especialmente significativas 
entre la altitud media que alcanzan estas masas ahuecadas si atendemos a la especie arbórea principal, es decir, encinas, robles rebollos, quejigos, alcornoques y fresnos.

Pero la importancia relativa que tiene cada una de estas especies sí varía considerablemente; los encinares adehesados suponen algo más del $65 \%$ del total de estos montes y los robledales de Quercus pyrenaica con casi un $28 \%$ son el otro gran conjunto. Por el contrario, los alcornocales tienen una presencia muy modesta, que no llega a las 3.000 ha, siempre según los datos que hemos podido extraer del Mapa Forestal de España.

Las dehesas se asientan en general sobre zonas bastante llanas, y de hecho la pendiente media que hemos podido calcular para el conjunto de estos terrenos es del 4.6\%; es verdad que puntualmente nos podemos encontrar con laderas bastante escarpadas, pero con pendientes superiores al $20 \%$ sólo hemos contabilizado 2.667 ha, y por el contrario algo más del $82 \%$ de estos montes no superan el $7 \%$ de pendiente. Resulta en consecuencia evidente que no parece que ni en el origen ni en el mantenimiento de estos paisajes haya tenido una influencia significativa el factor topográfico.

El sustrato sobre el que están situadas las dehesas es muy variado. Así, de las 65 unidades definidas por el Mapa Geológico de Castilla y León (SIEMCALSA, 1997) son 52 las que hemos podido identificar como soporte de estos montes. No obstante, la consideración de muchas de estas unidades puede soslayarse por su carácter episódico ( 22 de ellas no suman individualmente ni tan siquiera 500 ha, y la superficie de las 22 tan sólo supone el $0.67 \%$ del total), y eso es especialmente claro en lo que respecta al Mesozoico, que reúne poco más de 2.400 ha, la mayoría en la provincia segoviana. Con todo, no es tan apabullante como cabría pensar el peso de los terrenos típicos del zócalo, es decir, granitos y esquistos, ya que el terciario detrítico y los terrenos aluviales tienen una presencia consistente, sobre todo por las dehesas que se encuentran a lo largo del Corredor de Ciudad Rodrigo y en los bordes del resto del zócalo (ver GRÁFICO 1).

Puede sorprender que los terrenos sedimentarios cenozoicos y cuaternarios supongan algo más del $40 \%$ de la superficie ocupada por estos oquedales, pero hay que tener en cuenta que como comentábamos más arriba la mayor parte de las dehesas con este tipo de sustrato se encuentran en las proximidades del zócalo y presentan una continuidad natural con las que se asientan sobre granitoides y pizarras. En cualquier caso esto vuelve a poner de manifiesto que en el origen de estos paisajes no han sido los condicionantes naturales ni los únicos ni probablemente los factores más determinantes. 
Gráfico 1: Reparto de las principales unidades geolitológicas.

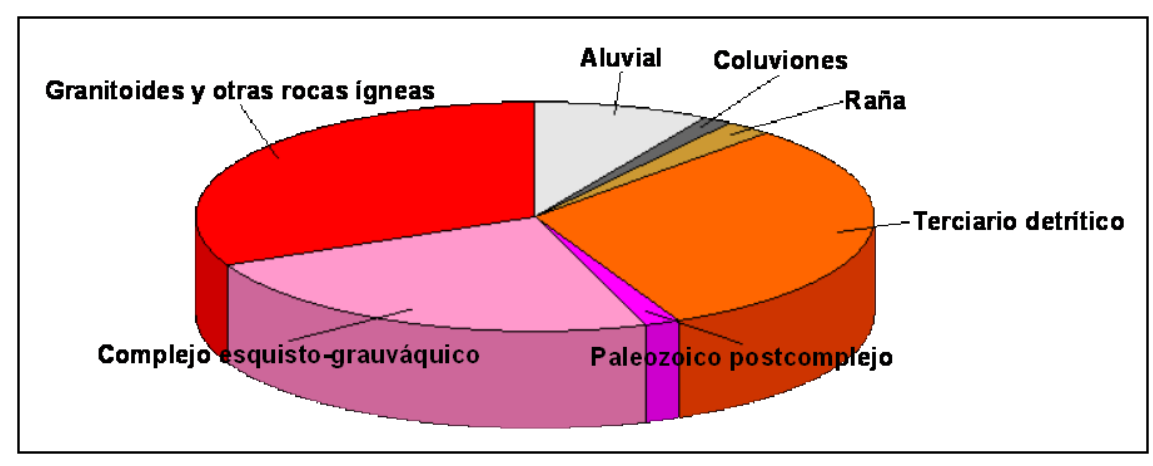

Sí se aprecian contrastes en las aparentes preferencias por los distintos sustratos por parte de las principales especies arbóreas de estos montes. Así, tanto quejigales como alcornocales son las masas que presentan unas inclinaciones más marcadas, en este caso por el Terciario terrígeno, y muy mayoritariamente (el $71 \%$ y el $80 \%$ respectivamente) vegetan sobre ese tipo de terrenos. Los encinares son, al contrario y como cabía esperar, los montes que tienen una distribución más equilibrada respecto de los valores medios y la única anomalía positiva que merece la pena reseñarse es la del Complejo esquisto-grauváquico. Los rebollares se asocian frecuentemente a los terrenos graníticos, y lo mismo podemos decir de las fresnedas, si bien en este caso hay que añadir la querencia de estas masas por los terrenos aluviales y de hecho el $30 \%$ de las fresnedas se sitúan sobre terrazas fluviales o el aluvial reciente.

Por comparación con las dehesas más meridionales, las explotaciones de este tipo en Castilla y León siempre se han distinguido en general por un invierno más riguroso, que implica en mayor o menor medida una parada vegetativa en esta estación. Si utilizamos el criterio de Gaussen según el cual se pueden considerar meses activos para la vegetación aquellos con temperatura media igual o superior a $6^{\circ} \mathrm{C}$, encontramos que al menos dos meses (diciembre y enero) presentan para el conjunto de la zona considerada un valor promedio inferior a $\operatorname{los} 6^{\circ}\left(5.1^{\circ}\right.$ y $4.3^{\circ}$ respectivamente $)$. En realidad, algo menos del $2 \%$ de las dehesas no presentan esa parada vegetativa invernal, y esta situación excepcional vuelven a protagonizarla las fincas del Valle del Tiétar.

La temperatura media anual de las dehesas es de $12.1^{\circ} \mathrm{C}$ y a este respecto no existen diferencias apreciables entre los distintos montes si atendemos a la especie arbórea dominante; otra cosa es si consideramos el rango de temperaturas, ya que en ese caso la horquilla es tanto más amplia cuanto mayor 
sea la extensión ocupada por cada una de las cinco especies consideradas, de manera que sería aventurado establecer algún tipo de relación causal sobre este particular. En cualquier caso el histograma que representa la extensión ocupada por los montes adehesados en diferentes intervalos de temperaturas medias anuales es suficientemente explícito sobre lo que resulta más común y también sobre lo que tiene un carácter más singular (ver GRÁFICO 2).

El monto anual de precipitaciones que reciben los montes adehesados tiene un valor medio de $563 \mathrm{~mm}$, y la variabilidad no es demasiado acentuada si consideramos que alrededor del $80 \%$ de las explotaciones se sitúan en el rango de entre los 450 y los $650 \mathrm{~mm}$. Con más de $950 \mathrm{~mm}$ y con menos de $350 \mathrm{~mm}$ sólo encontramos al $0.5 \%$ de estos montes. Las fresnedas y los rebollares son las masas que reúnen aportaciones más cuantiosas.

Gráfico 2: Superficie ocupada por los montes adehesados por intervalos de temperaturas medias anuales.

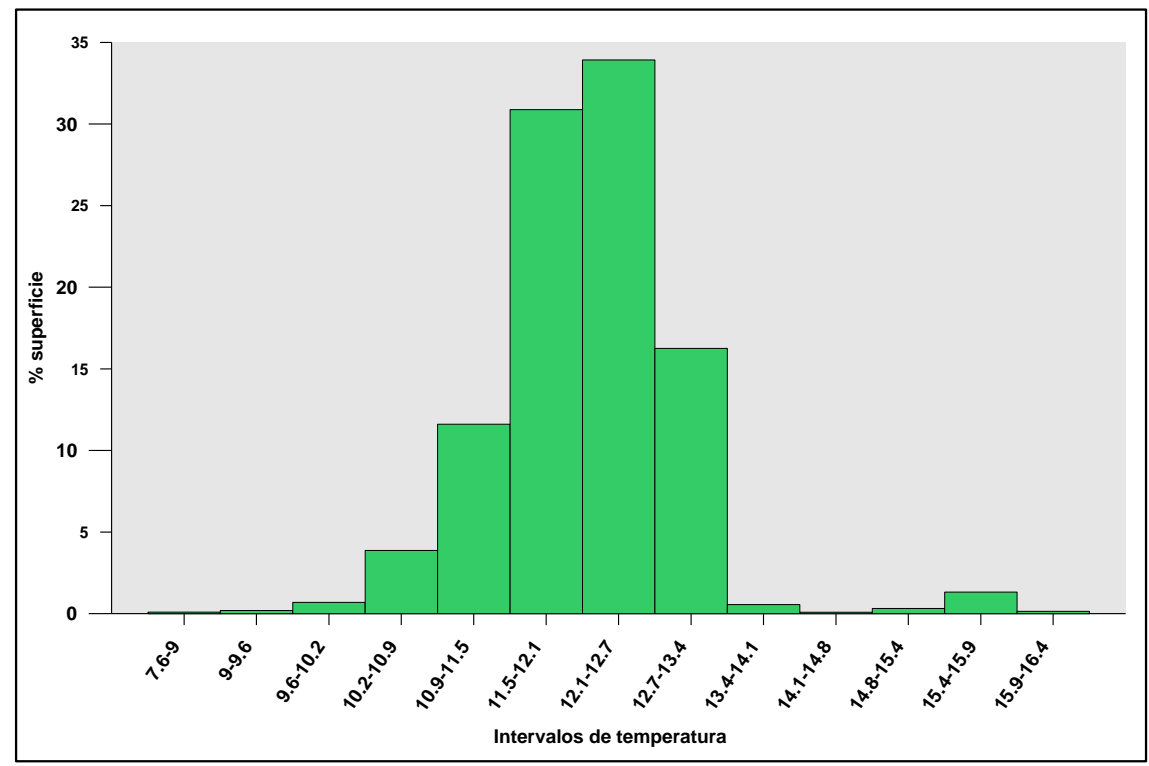

Sin embargo el rasgo más importante relacionado con la disponibilidad de agua tiene que ver con la marcada sequía veraniega; esta circunstancia condiciona especialmente los ciclos de aprovechamiento en las fincas y también obviamente el potencial productivo. Cuando además los suelos son escasamente profundos, los pastos se agostan rápidamente a causa de la mediocre capacidad de retención de agua. Hay que tener en cuenta que en julio la cantidad media de lluvia caída es de $15 \mathrm{~mm}$ (valores superiores a $30 \mathrm{~mm}$ sólo los reciben el $0.25 \%$ de los montes adehesados) y para el conjunto de los 
meses de julio, agosto y septiembre las precipitaciones suman en torno a 60 $\mathrm{mm}$. En definitiva esta es una característica común a las distintas áreas de dehesas de Castilla y León, e implica la aparición de una parada vegetativa estival generalizada, aunque la profundidad de la misma varíe de unas zonas a otras.

Con estos datos podemos estimar que la productividad potencial de las dehesas ronda los $4.200 \mathrm{~kg}$ de materia seca por ha y año (el rango de valores en el que se situaría el $88 \%$ de las fincas estaría entre los $3.500 \mathrm{~kg}$ y los $5.100 \mathrm{~kg}$ ), pero muy concentrados al final de la primavera en lo que respecta sobre todo a la producción de pasto, ya que en el otoño el pasto generado no llega a suponer habitualmente ni tan siquiera el $10 \%$ del total (VV.AA. 1992). Una vez más son las dehesas del Tiétar las que se presentan como un caso singular, ya que en esas fincas no se produce por término medio parón vegetativo en el invierno y la producción otoñal es más alta de la indicada más arriba; por todo ello son también las que presentan los índices de productividad más elevados ${ }^{2}$.

\section{TAMAÑO DE LAS EXPLOTACIONES Y RÉGIMEN DE TE- NENCIA}

Es habitual admitir que las dehesas son fincas grandes. Y es así. Pero también es verdad que podemos encontrarnos con tamaños bastante diversos, y aquí el problema principal suele residir en la definición de la talla mínima de lo que puede considerarse una explotación de este tipo. La muestra con la que nosotros hemos trabajado y que contrastaremos con otras fuentes (MARM 2008) consta de 1071 explotaciones que no incluyen los montes adehesados de aprovechamiento colectivo (como pueden ser determinados Montes de Utilidad Pública). La finca más pequeña que hemos identificado tiene 39 ha y la de mayor extensión cuenta con 3839 ha. El tamaño medio es, así, de 387 ha, pero las diferencias de dimensión son notablemente acusadas, de ahí que el coeficiente de variación sea algo superior al $90 \%$.

Este valor promedio contrasta con el que hemos calculado a partir del citado estudio del Ministerio. Según esta última fuente la dimensión media podría situarse en 247 ha, y eso una vez descontadas las explotaciones más pequeñas, lo que supondría que en Castilla y León existen algo más de 2100 empresas agrarias tipo dehesa. Las discrepancias considerables que hay entre

\footnotetext{
${ }^{2}$ La productividad potencial se ha estimado a partir de la evapotranspiración real anual. Y los valores calculados son claramente superiores a los rendimientos medidos de producción de pasto (VV.AA. 1992: 489-506), que en cualquier caso presentan una considerable variabilidad interanual y dentro de cada finca.
} 
las dos fuentes utilizadas y que pueden observarse en la TABLA $\mathrm{n}^{\circ} 2$ se deben seguramente a las diferencias en la manera de extraer la información, pero también hay que explicarlas por el tiempo transcurrido desde la elaboración de las Fichas de conservación a mediados del siglo pasado hasta la actualidad, nada menos que diez lustros. Cabe pues pensar que se ha producido en estas décadas una división de bastantes propiedades para dar lugar a explotaciones algo más pequeñas, pero esta es una hipótesis aventurada. Y decimos esto porque si se tienen en cuenta los datos de los Censos Agrarios de 1960 y 1999 en las zonas de más alta densidad de dehesas, se observa que se ha incrementado el número de las explotaciones más grandes en todos los tramos; es verdad que eso ha dado lugar paradójicamente a un leve descenso en el tamaño medio de las explotaciones, pero esa bajada no es comparable a la divergencia que hemos señalado entre las dos fuentes.

Tabla 2: Distribución porcentual del número y superficie de las dehesas según tamaños.

\begin{tabular}{|c|c|c|c|c|}
\hline \multirow{2}{*}{ Tamaños } & \multicolumn{2}{|c|}{ Fichas de conservación } & \multicolumn{2}{|c|}{ MARM, 2008} \\
\hline & \% del número & \% de la superf. & \% del número & \% de la superf. \\
\hline$<100$ ha & 10.83 & 2.11 & 10.81 & 3.16 \\
\hline $100-150$ ha & 12.04 & 3.93 & 29.77 & 14.79 \\
\hline $150-200$ ha & 11.11 & 4.98 & 16.99 & 11.85 \\
\hline 200-300 ha & 17.93 & 11.49 & 18.54 & 18.29 \\
\hline $300-500$ ha & 23.15 & 22.85 & 15.03 & 22.99 \\
\hline $500-1000$ ha & 19.14 & 33.39 & 7.35 & 19.50 \\
\hline$>1000$ ha & 5.79 & 21.25 & 1.5 & 9.42 \\
\hline
\end{tabular}

FUENTE: Fichas de conservación y MARM, 2008.

Las dehesas también se han identificado tradicionalmente por algunas notas relativas a las prestaciones laborales y al régimen de tenencia. Se daba así por hecho el carácter generalmente absentista de los propietarios y el importante peso que tenía en estas fincas el trabajo asalariado. En realidad, en esto como en tantas otras cosas existía y existe una tipología bastante variada y que obedece a factores muy diversos. Es verdad que algunos profesionales de éxito se han convertido en propietarios de dehesas por diversas motivaciones: como inversión, para obtener una "renta ambiental" o por razones ligadas al prestigio social; igualmente es cierto que algunos propietarios de dehesas que heredaron estas fincas obtienen sus principales ingresos por actividades que poco tienen que ver con el sector agropecuario. Pero igualmente es indudable que se ha producido en las últimas décadas un incremento en la profesionalización en la gestión de estas explotaciones, lo que se ha reflejado en mayores inversiones (en cerramientos especialmente, pero también en otras instalaciones, en el propio ganado, en maquinaria,...) y en procesos de racionalización. Así, cada vez son menos frecuentes los casos de fincas en 
que aparecen disociados los distintos aprovechamientos $\mathrm{y}$, por lo tanto, su gestión. La explotación, sea cual sea el régimen de tenencia, se lleva como un todo, lo que obviamente resulta más coherente con el tipo de aprovechamiento integrado característico de las dehesas.

Establecer la situación y la evolución del régimen de tenencia en las dehesas resulta complicado, ya que como es sabido los datos del Censo Agrario no singularizan la información referida a tipos específicos de sistemas agrarios como el que nos ocupa. Así los datos que nosotros hemos extraído tienen un carácter aproximativo y además se refieren a una muestra. En concreto, hemos establecido los porcentajes sobre la superficie total asignados a cada régimen de tenencia en 64 municipios de la región en los que la superficie ocupada por dehesas supera el $60 \%$ del término municipal. Los datos los presentamos en la tabla 3. Se aprecia que hasta el último año censal venía creciendo el porcentaje de terreno llevado en propiedad, que siempre aparece como el régimen mayoritario, aunque no con el alcance que proporcionan otras fuentes de información. Por ejemplo, en una encuesta realizada para el conjunto de España se concluye que el $80 \%$ de estas explotaciones se llevan en propiedad, y el $65 \%$ de los titulares del total de fincas declaran que la mayor parte de sus ingresos provienen de las explotaciones (MARM, 2008).

Aunque se ha producido un incremento relativo de las prestaciones laborales llevadas a cabo por los dueños, y eso dentro de un marco general de reducción de fuerza de trabajo, lo cierto es que el papel de los propietarios de dehesas que llevan su explotación presenta algunas variaciones, y así es posible establecer una tipología muy simple que se basa fundamentalmente en el tamaño de las fincas: a) los propietarios de dehesas más pequeñas trabajan directamente la explotación además de llevar las labores de gestión o dirección; su origen suele ser local y pueden haber heredado las tierras o ser antiguos renteros de dehesas que acabaron comprando; suelen vivir en las propias fincas o en los núcleos rurales más próximos; b) los propietarios medianos tienen básicamente funciones de dirección, comerciales y de gestión y no tanto de trabajo directo en la finca; habitualmente viven en núcleos urbanos relativamente próximos a la explotación y en ocasiones tienen rentas procedentes de otras actividades económicas; c) por último, con frecuencia los grandes propietarios explotan las fincas bajo formas societarias que además no tienen en una única explotación su principal fuente de ingresos; la gestión de las dehesas no la suelen llevar directamente los propietarios, sino profesionales contratados a tales efectos, y el lugar de residencia de estos propietarios suelen ser las principales ciudades del país.

Como comentábamos más arriba, las dehesas se llevan con una decreciente 
cantidad de mano de obra, que por esa razón tiene que tener un carácter polivalente. Por eso también en las tareas del campo se ha incrementado el protagonismo de los propietarios. En todo caso, en la actualidad estas fincas se caracterizan por una baja intensidad en el empleo de mano de obra (LLORENTE, 2008), y los asalariados siguen reuniendo la mayoría de las unidades de trabajo de estas explotaciones, ya que únicamente en torno al $20 \%$ de las dehesas carecen de empleados, y estimamos que el $72 \%$ de las UTA proceden de asalariados. Estos trabajadores suelen ser fijos, de manera que la temporalidad no es, en consecuencia, una característica del empleo en la dehesa.

Tabla 3: Porcentaje de la superficie total de cada tipo de régimen de tenencia en los muncicipios con más del 60\% del término ocupado por dehesas.

\begin{tabular}{crrrr} 
Año & Propiedad & Arrendamiento & Aparcería & Otros \\
\hline 1962 & 64.21 & 31.33 & 1.71 & 2.72 \\
1972 & 64.31 & 32.67 & 0.96 & 2.03 \\
1982 & 65.87 & 31.53 & 0.75 & 1.85 \\
1989 & 67.56 & 30.44 & 0.50 & 1.50 \\
1999 & 55.89 & 40.57 & 0.30 & 3.21 \\
\hline
\end{tabular}

FuENTE: Censos Agrarios.

\section{PAISAJES Y TRANSFORMACIONES EN EL SISTEMA DE APROVECHAMIENTO}

El mantenimiento o conservación del terreno adehesado a lo largo del tiempo ha tenido que ver sin duda desde el punto de vista histórico con sus peculiaridades patrimoniales; y, así, como ha ocurrido en otros lugares de la Península Ibérica, también aquí encontramos asociados gran propiedad (particular, pero también pública) y mantenimiento de paisajes o agrosistemas escasamente artificializados. Pero la dehesa no es sólo una finca de ciertas dimensiones, sino que es especialmente un sistema de aprovechamiento con una lógica básica que se expresa en diferentes unidades de paisaje y funcionales, aunque sea el monte abierto con diferentes grados de densidad la imagen sintética de este agrosistema. Y aunque resulta complicado hablar de un tipo o modelo de dehesa, en función no sólo de los contrastes entre unas áreas y otras sino también si atendemos a los cambios que han experimentado estas fincas, también es verdad que existen unas características razonablemente generales.

La lógica de este estilo de aprovechamiento se fundamenta en lograr un compromiso entre el crecimiento y la estabilidad. Y eso porque las condiciones ambientales en las que se han desarrollado estos paisajes no han aconsejado nunca fórmulas de explotación más intensivas. Es así como nos encon- 
tramos en los terrenos adehesados esa dualidad, que pretende ser equilibrio, entre elementos maduros, con una productividad por lo general limitada, pero de gran estabilidad y con una notable acumulación de biomasa, y elementos jóvenes de una estabilidad menor pero mayor productividad. La vegetación leñosa representa esos elementos maduros, mientras que el pastizal o, eventualmente, los cultivos se configuran como componentes capaces de una movilización más rápida y productiva de los recursos, pero también más vulnerables en los momentos de carestía y mucho más lábiles (VV.AA. 1992).

Esa búsqueda del equilibrio entre productividad y estabilidad se proyecta sobre el territorio con diferentes matices, ya que esa complementariedad agrosilvopastoral no se consigue siempre con el mismo tipo de combinación entre elementos maduros y jóvenes, de forma que nos encontramos con una cierta diversidad paisajística que es producto de los contrastes en el potencial ecológico pero también de las decisiones de los explotadores: manchas de monte cerrado, carrascales, montes ahuecados con diferentes grados de densidad arbórea, prados o pastizales sin árboles o casi sin monte, cultivos bajo monte o sin árboles,... (ver FIGURA 2).

Figura 2: Terrenos de cultivo, pastizal y monte en una dehesa.

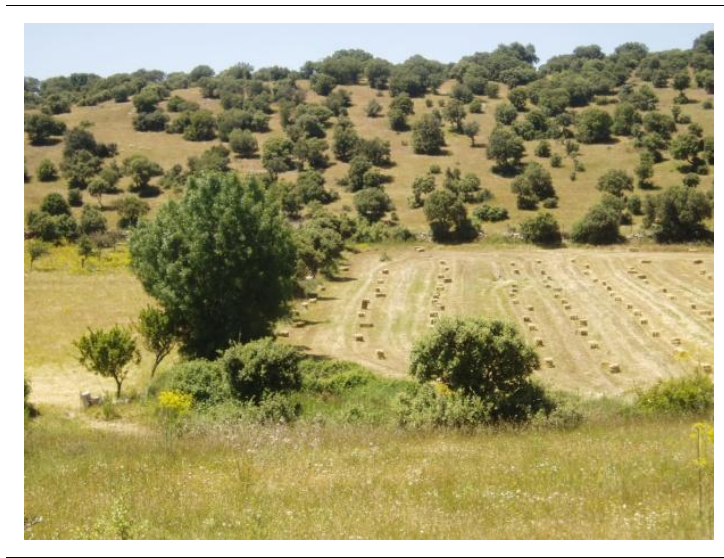

FuENTE: José Manuel Llorente Pinto, Junio de 2008, Horcajo Medianero, Salamanca

El adehesamiento se basa en un uso adecuado y combinado de esa dualidad y, por lo tanto, en la integración complementaria de los aprovechamientos agrícolas, forestales y pecuarios, siendo la orientación predominante la ganadera; por ello, la ordenación del monte y la gestión de los cultivos se tienen que subordinar a la cría de los ganados. Ahora bien, como el potencial ecológico con el que se cuenta proporciona unos recursos limitados en cantidad y sometidos a una fuerte estacionalidad, es necesario utilizar una fórmula que mitigue lo más posible esas irregularidades en la producción maximizando los reempleos y autoconsumos. Y así el cultivo, que eventualmente puede servir para controlar el matorral, proporciona granos, paja y forrajes con los que hacer frente a los baches productivos del invierno y del verano; en el ve- 
rano también se aprovechan los rastrojos y el ganado puede ramonear cuando ya no queda pasto o sólo hay pasto seco (ver figura 3).

$\mathrm{Y}$ ese es uno de los sentidos de la existencia de monte en estas fincas; obviamente el monte es útil para guarecer al ganado de las peores inclemencias meteorológicas, como comentábamos más arriba, pero además su efecto sobre el suelo y sobre las condiciones microclimáticas favorece la diversificación del pasto y alarga en el tiempo la producción del mismo; en fin el monte complementa directamente la alimentación del ganado

Figura 3: Vaca ramoneando durante el verano en un sector de monte espeso. en épocas de escasez gracias

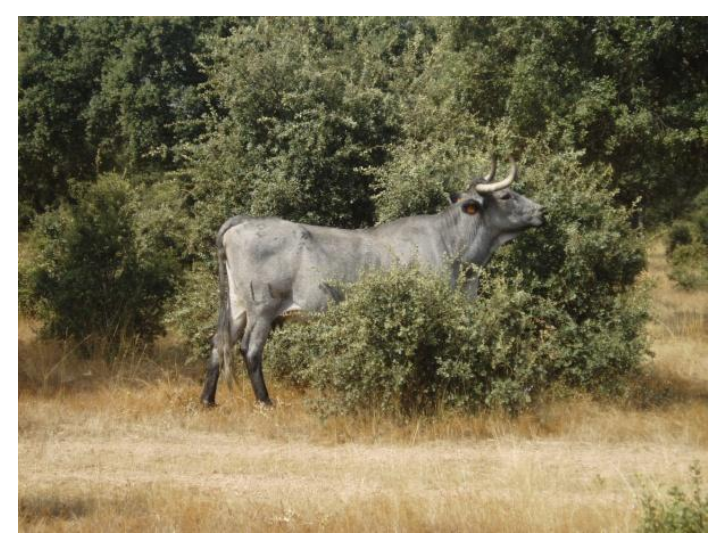

FuENTE: José Manuel Llorente Pinto, Agosto de 2002, Canillas de Abajo, Salamanca. al ramón y, especialmente, la bellota. Por todo esto el mantenimiento de un monte en buen estado resulta fundamental para el sostenimiento de este modelo de explotación.

El arbolado o la eventual presencia de matorral influyen en la calidad y cantidad de pasto, y lo mismo se puede decir de los rendimientos de los cultivos. En general el monte implica una disminución en la generación de hierba que es tanto más patente en términos relativos cuanto mayor sea el potencial productivo del terreno, de ahí que lo razonable sea que en las fincas aparezca un gradiente de densidad del arbolado inversamente proporcional a la productividad del suelo: el monte más abierto o incluso ausente se corresponde con las tierras de cultivo que siguen rotaciones cortas y con los pastizales que ocupan las riberas o las navas más húmedas; bajas o medias densidades aparecen en sectores de cultivo ocasional y en majadales; densidades de medias a altas son propias de los vaqueriles, y las mayores densidades son las lógicas en las áreas con mayores pendientes o con suelos más insuficientes.

El grado de cubierta resulta ser así en teoría un compromiso entre los beneficios y perjuicios que ocasiona el arbolado, y lo ideal consistiría en conseguir en cada sector de las fincas una combinación óptima. Pero además hay que tener en cuenta el propio dinamismo del monte y sus necesidades de regeneración; es decir, se suele tender en muchos cercados de las explotacio- 
nes adehesadas a obtener una especie de óptimo intemporal que sería algo así como una imagen caracterizada por grandes árboles aparasolados que con densidad media-baja salpican un pastizal totalmente desprovisto de matorral. Esta foto-fija de carácter finalista puede interpretarse como una suerte de fosilización que no debe perpetuarse indefinidamente (ver FIGURA 4), pues de lo contrario acabaría por descender peligrosamente el número de pies y por desaparecer el monte, muy especialmente por el incremento de la presión ganadera que se ha producido en las fincas.

El manejo tradicional del ganado se basaba en una cabaña bastante diversificada, en una densidad modesta limitada por los recursos pastables totales y muy especialmente por los baches productivos- y en una organización espacio-temporal bastante laxa, es decir, con controles escasos y frecuentemente limitados a las prioridades que se establecían en el aprovechamiento de determinados recursos. Pero esta situación ha cambiado

Figura 4: Monte nuevo de encinos pero sin renuevos.

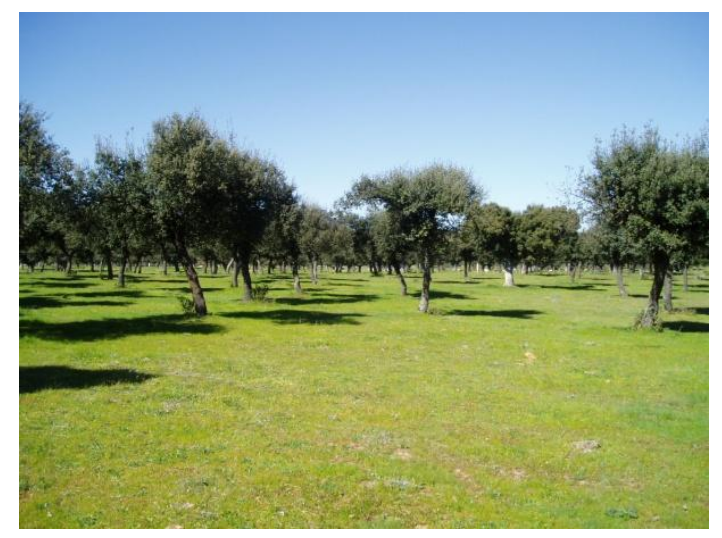

FuENTE: José Manuel Llorente Pinto, Abril de 2004, Tabera de Abajo, Salamanca. al compás de otras transformaciones (LLORENTE, 2008). En la actualidad la cabaña se ha simplificado a favor del porcino y del vacuno, pero sobre todo se ha incrementado muy notablemente y no sólo en número, sino que en general los animales son ahora mayores por la generalización del cruce industrial, lo que significa una presión añadida sobre los recursos. Si atendemos nuevamente a los 64 municipios con una superficie ocupada por dehesas superior al $60 \%$ del término, resulta que desde principios de los años 60 del siglo pasado hasta el año 2003 las unidades ganaderas agregadas de bovino, ovino, caprino y porcino se han cuadruplicado, pasando de 47.105 a 188.497 (147.201 U.G. si preferimos los datos del Censo Agrario de 1999) y eso sobre una superficie total de 337.945 ha, que es la extensión ocupada por esos 64 municipios.

El incremento ganadero ha sido bastante general, pero las dehesas han sido protagonistas esenciales de ese aumento, ya que entre las fechas señaladas más arriba y para el conjunto de Castilla y León las unidades ganaderas sumadas de vacuno, lanar, caprino y porcino se han duplicado, mientras que, 
como hemos comentado ya, en las dehesas se multiplicaban por cuatro. Y ese crecimiento se ha fundamentado en estas explotaciones en la multiplicación del vacuno (casi se ha sextuplicado en la muestra que hemos utilizado), y no sólo para la cría sino también para el cebo, y lo mismo ha ocurrido con el porcino ibérico, y esto de forma más explosiva, ya que los mayores incrementos se han producido en los últimos lustros, de tal manera que por cada cerdo que había a principios de los años sesenta en las dehesas hoy nos encontramos con cinco.

Mapa 2: Distribución de las vacas de orientación cárnica y de las ganaderías de toros bravos

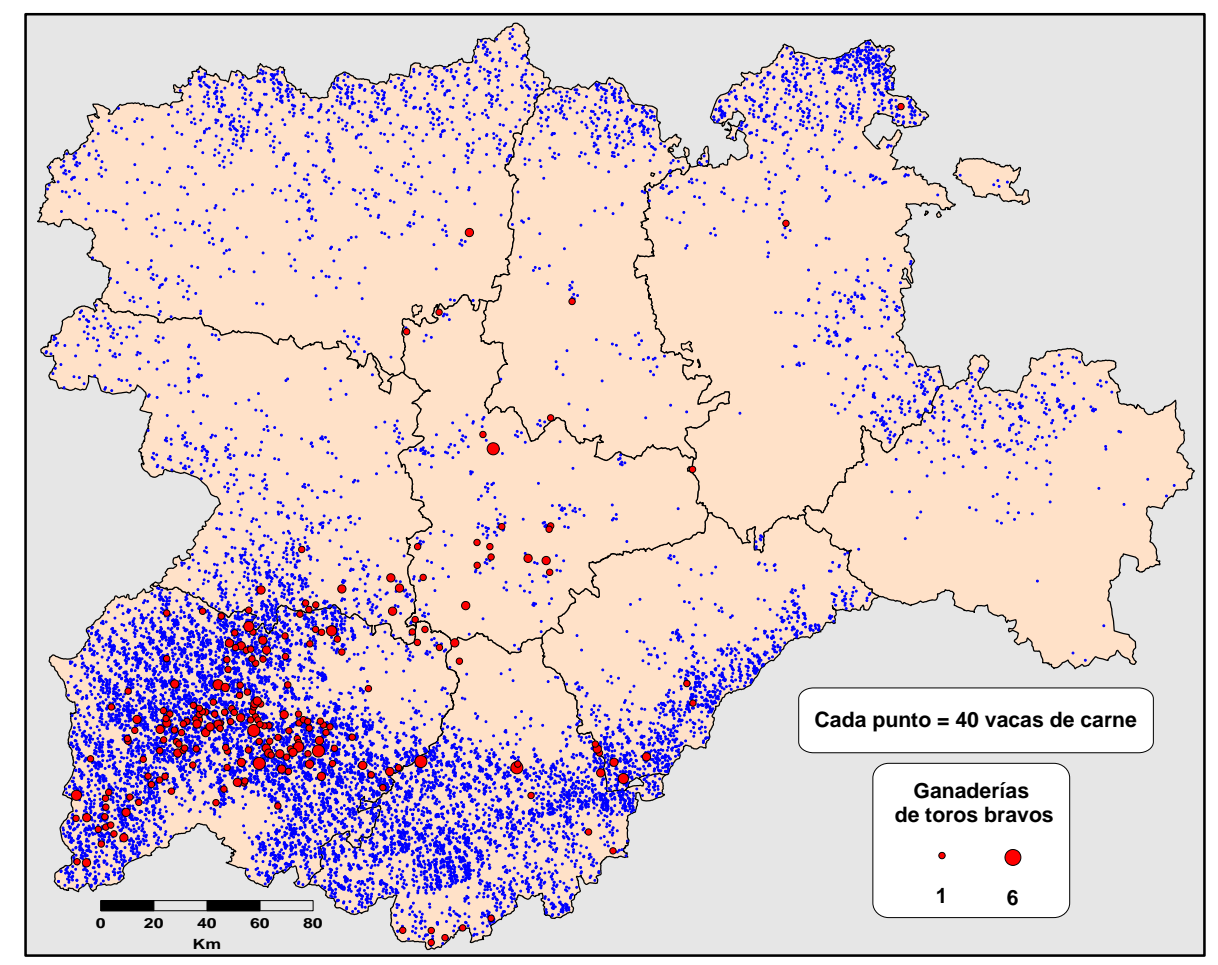

FUENTE: http://www.cetnotorolidia.es/opencms_wf/opencms/ganaderias/index.html, y Junta de Castilla y León, Censo Ganadero de 2003.

No puede extrañar por todo esto que si representamos en un mapa de la región las cabezas de ganado del vacuno de carne, sean las áreas adehesadas las que concentran buena parte de ese capital ganadero, y, como es lógico, también la distribución de las ganaderías de toros bravos se ajusta notablemente a las zonas de mayor densidad de dehesas (ver mapa 2). Pero lo más trascendente de este proceso de intensificación ganadera es el impacto que 
puede estar teniendo sobre el estilo de aprovechamiento y sobre las dehesas como sistemas agrarios.

Es evidente, como hemos visto, que estas fincas han tenido un enorme éxito aparente en su proceso de modernización, que se ha sustanciado en esos incrementos de densidad pecuaria. Eso ha sido posible porque las dehesas presentan sin duda unas ventajas competitivas para la cría de ganado frente a otro tipo de explotaciones. Tenían terreno y, por lo tanto, un margen considerable, por ejemplo, para incrementar esa carga de animales sin que nominalmente el régimen ganadero dejara de ser extensivo; disponían de una infraestructura básica y de bastante flexibilidad y contaban con una amplia experiencia en el manejo del ganado. Y así no sólo aumentaron las cargas con la idea de generar economías de escala, sino que también muchas explotaciones decidieron cerrar los ciclos productivos. Las dehesas dejaron de ser un simple ámbito de cría y se decidieron a cebar esos animales, especialmente terneros, que antes se enviaban a Aragón, Cataluña o Toledo.

Estas estrategias podían obedecer a circunstancias externas o internas a las fincas y podían considerarse más o menos forzadas y tener por lo mismo un carácter adaptativo; así la preferencia por el vacuno ha tenido mucho que ver con su mayor facilidad de manejo en un contexto de reducción de la mano de obra, pero también las demandas del mercado han sido trascendentes; en otras situaciones sin embargo las transformaciones se han visto claramente estimuladas por factores positivos. Ese es el caso de las primas ganaderas ligadas a la PAC desde hace varios lustros, que se han comportado como un importante acicate del proceso de intensificación al menos hasta el momento. Las dehesas se han vuelto así también muy dependientes de las subvenciones, ya que estimamos que por término medio el 35\% de la renta agraria de estas explotaciones proviene de las ayudas directas, y se ha estimado que el $8 \%$ de las dehesas (en general las más pequeñas) no obtendrían beneficios si desaparecieran las subvenciones (MARM, 2008: 342).

Este comentado incremento en la carga ganadera, la generalización del cruce industrial, las transformaciones en los ciclos productivos y las exigencias del mercado y de la administración en lo referente tanto a rendimientos cárnicos como a sanidad animal han hecho de las fincas sistemas menos autosuficientes, más dependientes de los insumos externos, menos eficaces en la gestión de sus recursos, menos equilibrados y más inestables. Como la alimentación complementaria se ha incrementado mucho y se suele ofrecer al ganado en los mismos lugares de la finca, frecuentemente los más accesibles, el ganado no se ve obligado a aprovechar otros recursos que antes utilizaba y en muchas explotaciones se aprecian sectores con exceso de pisoteo o frecuentación y otros, los más excéntricos o los menos apetecibles, en los 
que el monte cada vez se espesa más. Y ambas situaciones extremas son perniciosas.

De ahí la creciente preocupación que hay por conseguir que estos montes adehesados permanezcan en buen estado. Y para ello se debe partir de estas bases: por un lado, lograr y mantener densidades apropiadas en cada lugar, proceder a un manejo adecuado de esas masas mediante el aclarado, encabezado de encinos, olivo y poda, y, además, asegurar las posibilidades de regeneración en aquellos sectores con pies más viejos y menor densidad. La escasa regeneración es probablemente uno de los problemas más acuciantes y que puede comprometer de manera más radical el futuro de las dehesas; aunque es verdad que en Castilla y León la situación no es tan grave como en Andalucía o Extremadura, lo cierto es que según el Tercer Inventario Forestal Nacional algo así como el $40 \%$ de la superficie de las dehesas de esta región no muestra regeneración.

\section{CONCLUSIONES}

Las dehesas representan un buen ejemplo de un tipo de explotación equilibrada de los recursos en ambientes limitados por un potencial ecológico insuficiente. En este contexto, la estrategia de gestión se fundamenta en optimizar el aprovechamiento de la productividad del territorio, logrando unos rendimientos sostenidos. Y este proceso de optimización tiene tres bases: la diversificación productiva, la complementariedad entre los distintos usos y la extensificación en la utilización de los recursos naturales.

Con esos pilares se puede alcanzar un razonable equilibrio y además conseguir un sistema agrario que presente unos importantes valores ambientales. Sin embargo, las transformaciones socioeconómicas de las últimas décadas han trastocado esas bases y están sometiendo a estas fincas a una especie de prueba de esfuerzo. La adaptación a esos cambios ya ha comenzado a dejar efectos patentes sobre los paisajes, a pesar de la enorme inercia que presentan estos montes adehesados, y así las nuevas realidades significan un duro examen para ese armónico estilo de aprovechamiento. Tanto es así que para impedir o frenar el deterioro de algunas zonas empieza a verse la necesidad de adoptar algunas medidas.

En algunos casos, ha sido la propia PAC la que ha estimulado la intensificación ganadera, pero al tiempo es cierto que si no fuera por esas subvenciones muchas dehesas se encontrarían en una situación incierta. En consecuencia, uno de los más importantes desafíos consiste en tratar de ofrecer un sostén a las explotaciones que diera prioridad al equilibrio del propio sistema; esto consistiría en identificar aquellas peculiaridades de las dehesas que pre- 
sentan más fragilidad y orientar las ayudas hacia los aspectos o sectores que apoyen el mantenimiento funcional de estas fincas sin comprometer su esencia, y, al contrario, evitar aquellos incentivos que favorecen los desequilibrios.

Hay que decir en todo caso que la modernidad no sólo ha supuesto una amenaza para la pervivencia de la dehesa, también es preciso que resaltemos que existen nuevos escenarios, nuevos marcos vitales y conceptuales, que funcionan a favor de una perdurabilidad razonablemente armónica de los sistemas agrosilvopastorales. Nos referimos, por ejemplo, a la creciente demanda de productos singularizados y de calidad, pero también al progresivo valor que se le adjudica a las funciones ambientales en cualquier sistema agrario y a la gradual utilidad que van a adquirir las fincas que sean más eficientes y menos despilfarradoras en el uso de los recursos. Todo esto puede suponer un acicate para una gestión más cuidadosa, más integrada y más racional de las dehesas.

En todo caso, a largo plazo la conservación no fosilizada de estos paisajes agrarios sólo será posible a través de una mayor atención al arbolado; hay que entender que no sólo las producciones más interesantes y apreciadas de la dehesa (las procedentes del cerdo ibérico) sino su propia configuración y funcionamiento dependen del monte; pero ha sido el monte probablemente el subsistema que ha sufrido las mayores perturbaciones en los últimos lustros, lo que se manifiesta en un estado sanitario mediocre en muchos casos y, sobre todo, en el envejecimiento, un aclarado excesivo en algunos sectores y un cierto abandono en otros. La sobrecarga de ganado que se aprecia desde hace unos años y el manejo de ese ganado afectan a la regeneración y al estado general del monte. En consecuencia, un mayor esmero en el cuidado del arbolado y una ordenación más adecuada del pastoreo con cargas más ajustadas a las posibilidades de las fincas parecen los retos más importantes a los que se enfrentan estas explotaciones los próximos años.

\section{BIBLIOGRAFÍA}

Alario Trigueros, M. Ta; Delgado Huertos, E.; Dueñas Cepeda, $M^{a}$ J. y García Colmenares, P. (1981): «Cambios de uso y formación vegetal en el espacio del Monte "El Viejo" de Palencia». Publicaciones de la Institución Tello Téllez de Meneses, $\mathrm{N}^{\circ}$ 45, 57-131.

Llorente PinTO, J. M. (1985): Los paisajes adehesados salmantinos. Salamanca, Centro de Estudios Salmantinos.

Llorente Pinto, J. M. (1995): «La dehesa salmantina». Medio Ambiente en Castilla y León. $\mathrm{N}^{\circ}$ 4, 26-34.

LLORENTE PinTo, J. M. (2008): «Cambios y permanencias en los sistemas adehesados. El caso de las dehesas salmantinas». ERÍA. $\mathrm{N}^{\circ} 77$, 315-330. 
MARM (2008): Diagnóstico de las dehesas ibéricas mediterráneas. 766 En línea: http://www.mma.es/portal/secciones/biodiversidad/montes_politica forestal/sani dad forestal/pdf/anexo 3 4 coruche 2010.pdf, consultado el 02/11/2010.

Mata Olmo, R. (2002): «Paisajes y sistemas agrarios de España» en Agricultura y Sociedad en el cambio de siglo (GómEZ BENITO, C. y GonZÁlez, J.J., coor.). Madrid, McGraw Hill, 3-62.

Pulido, F. y PiCARDo, A. (Coor.) (2010): Libro Verde de la Dehesa. 48 En línea: http://www.uco.es/integraldehesa/images/stories/doc/Jornadas/libro_verde_ dehesa.pdf, consultado el 04/11/2010.

RAMOS SANTOS, J.M ${ }^{\mathrm{a}}$ (2008): «Las transformaciones de los montes en la época de las desamortizaciones en el interior de la Cuenca del Duero». Boletín de la AGE. $\mathrm{N}^{\mathrm{o}} 46,305-327$.

SÁNCHEZ GÓMEZ, L.A. (1992): «Evolución histórica de la dehesa como sistema de apropiación y explotación de los recursos naturales. El ejemplo de la comarca de Sayazo (1752-1992)». Agricultura y Sociedad. № 73, 221-246.

SiEMCALSA (1997): Mapa Geológico y Minero de Castilla y León, Escala 1/400.000, Valladolid, Junta de Castilla y León.

VV.AA. (1992): El libro de las dehesas salmantinas. Salamanca, Junta de Castilla y León. 